\title{
Electric-magnetic black hole duality
}

\author{
S. Deser \\ Department of Physics, Brandeis University, Waltham, Massachusetts 02254
}

M. Henneaux

Faculté des Sciences, Université Libre de Bruxelles, Campus Plaine C. P. 231, B-1050, Bruxelles, Belgium and Centro de Estudios Científicos de Santiago, Casilla 16443, Santiago, Chile

C. Teitelboim

Centro de Estudios Científicos de Santiago, Casilla 16443, Santiago, Chile and School of Natural Sciences, Institute for Advanced Study, Princeton, New Jersey 08540

(Received 19 July 1996)

\begin{abstract}
We generalize duality invariance for the free Maxwell action in an arbitrary background geometry to include the presence of electric and magnetic charges. In particular, it follows that the actions of equally charged electric and magnetic black holes are equal. [S0556-2821(97)02602-7]
\end{abstract}

PACS number(s): 04.70.Bw, 04.20.Cv, 04.20.Fy, 11.30.Ly

It has long been known [1] that in an arbitrary background geometry, the source-free Maxwell action is duality-invariant under general electric/magnetic field rotations (for a recent review of electromagnetic duality, see [2]). This is the case despite the change of sign of the Maxwell Lagrangian $-\frac{1}{4} F_{\mu \nu}^{2}$ under the "duality rotation" $F_{\mu \nu} \rightarrow{ }^{*} F_{\mu \nu}$. Because of the interest of electrically and magnetically charged black holes in the semiclassical regime, it is natural to study duality of the action there as well. We shall show that the action, and not just the field equations or the stress tensor, is duality invariant in such a way that equivalence of the black hole actions and partition functions is maintained for the charged solutions. In particular, we provide a simple derivation of similar recent conclusions of Hawking and Ross [3], which were reached through a somewhat laborious procedure. As the subject of Maxwell duality seems always to be fraught with confusion, we will at times belabor the obvious to make our point clear.

When dealing with magnetically charged black holes, one usually determines the partition function in a sector with fixed magnetic charge. To make a meaningful comparison between the electric and magnetic cases, it is therefore necessary to compute the partition function for electrically charged black holes also in a sector with fixed charge, rather than fixed electric chemical potential. For this reason, we shall deal in this paper exclusively with the action adapted to the variational principle in which the charges are kept fixed. Technically, this is achieved by considering field histories in the variational principle that all have the same given electric and magnetic fluxes at infinity (see below). Our main result is that there is a transformation of the dynamical variables which, when combined with the corresponding rotation of the charges, maps the action for a fixed set of electric and magnetic charges to the action for the duality-rotated set of fixed electric and magnetic charges.

Consider first sourcefree electrodynamics in an arbitrary background with topology $R \times \Sigma$, where the spatial sections $\Sigma$ are homeomorphic to $R^{3}$. As shown explicitly in [1], the
Maxwell action is invariant under arbitrary finite duality rotations. In canonical form, in the usual $3+1$ notation, it is [4]

$$
\begin{aligned}
I_{M}[\mathbf{E}, \mathbf{A}]= & -\int d^{4} x\left[E^{i} \dot{A}_{i}+\frac{1}{2} N g^{-1 / 2} g_{i j}\left(E^{i} E^{j}+B^{i} B^{j}\right)\right. \\
& \left.-\epsilon_{i j k} N^{i} E^{j} B^{k}\right],
\end{aligned}
$$

where $E^{i}$ is the electric, $B^{i} \equiv \epsilon^{i j k} \partial_{j} A_{k}$ the magnetic field (both are contravariant three-densities), and all metrics are in three-space; we have solved the Gauss constraint so that both $E^{i}$ and $B^{i}$ are identically transverse, $\partial_{i} E^{i}=0=\partial_{i} B^{i}$. As usual, we take the field configurations to behave asymptotically as $A_{i}=a_{i}(\theta, \phi) r^{-1}+O\left(r^{-2}\right) \quad$ and $E^{i}=e^{i}(\theta, \phi) r^{-2}+O\left(r^{-3}\right)$ as $r \rightarrow \infty$.

The variation of the action under changes of $E^{i}$,

$$
\delta_{E} I_{M}=-\int d^{4} x \delta E^{i}\left(\dot{A}_{i}+N g^{-1 / 2} g_{i j} E^{j}-\epsilon_{i j k} B^{j} N^{k}\right)
$$

vanishes for arbitrary variations $\delta E^{i}$ subject to the transversality conditions ${ }^{1} \partial_{i} \delta E^{i}=0$ and $\delta \oint_{S_{\infty}^{2}} E^{i} d S_{i}=0$ if and only if the coefficient of $\delta E^{i}$ in (2) satisfies the condition

$$
\dot{A}_{i}+N g^{-1 / 2} g_{i j} E^{j}-\epsilon_{i j k} B^{j} N^{k}=\partial_{i} V
$$

where $V\left(\equiv A_{0}\right)$ is an arbitrary function which behaves asymptotically as $C+O\left(r^{-1}\right)$. In that case, $\delta I_{M}$ $=-\int d^{4} x \delta E^{i} \partial_{i} V=-\oint_{S_{\infty}^{2}} \delta E^{i} V d S_{i}=-C \delta($ electric flux $)=0$. No special conditions are required, on the other hand, when varying $A_{i}$. Thus, the action (1) is appropriate as it stands, i.e., without "improving'" it by adding surface terms, to the

\footnotetext{
${ }^{1}$ The condition $\delta \oint_{S_{\infty}^{2}} E^{i} d S_{i}=0$ is actually a consequence of $\partial_{i} \delta E^{i}=0$ (and of smoothness) on spatial sections with $R^{3}$ topology. We write it separately, however, because this is no longer the case if $\Sigma$ has holes, as below.
} 
variational principle in which the competing histories all have the same given electric flux at infinity and thus also the same given electric charge (here equal to zero).

As pointed out in [3], it is necessary to allow the temporal component $V$ of the vector potential to approach a nonvanishing constant at infinity since this is what happens in the black hole case if $V$ is required to be regular on the horizon. However, as we have just shown, in order to achieve this while working with the action (1), it is unnecessary to keep all three components $E^{i}$ of the electric field fixed at spatial infinity; only the electric flux $\oint_{S_{\infty}^{2}} E^{i} d S_{i}$ must be kept constant in the variational principle.

The action (1) is invariant under duality rotations. Indeed, the finite rotation

$$
\left(\begin{array}{l}
\mathbf{E}^{\prime} \\
\mathbf{B}^{\prime}
\end{array}\right)=R\left(\begin{array}{l}
\mathbf{E} \\
\mathbf{B}
\end{array}\right) \equiv\left(\begin{array}{cc}
\cos \theta & \sin \theta \\
-\sin \theta & \cos \theta
\end{array}\right)\left(\begin{array}{l}
\mathbf{E} \\
\mathbf{B}
\end{array}\right)
$$

manifestly preserves all but the EAं term in Eq. (1), whose separate invariance is also easy to check. The above rotation of $\mathbf{B}$ can of course be explicitly expressed as one of $\mathbf{A}$ using the fact that $A^{i}=-\nabla^{-2} \epsilon^{i j k} \partial_{j} B_{k}$ up to an irrelevant gauge transformation. A particularly symmetric formulation results if one introduces, as in [1], a "potential" $\mathbf{Z}$ for the transverse electric field through $E^{i}=\epsilon^{i j k} \partial_{j} Z_{k}$. The equivalent of Eq. (4) is then

$$
\left(\begin{array}{l}
\mathbf{Z}^{\prime} \\
\mathbf{A}^{\prime}
\end{array}\right)=R\left(\begin{array}{l}
\mathbf{Z} \\
\mathbf{A}
\end{array}\right) \equiv\left(\begin{array}{cc}
\cos \theta & \sin \theta \\
-\sin \theta & \cos \theta
\end{array}\right)\left(\begin{array}{l}
\mathbf{Z} \\
\mathbf{A}
\end{array}\right)
$$

and the kinetic term

$$
\int d^{4} x \epsilon^{i j k} \partial_{j} Z_{k} \dot{A}_{i}
$$

is now manifestly invariant. (The surface term that one picks up at spatial infinity from the variation of the kinetic term is easily seen to vanish with the given asymptotic conditions.) As explained in [1], there is no contradiction between the invariance of the action (1) under (4) and its change of sign under

$$
F_{\mu \nu} \rightarrow(-g)^{-1 / 2} g_{\mu \alpha} g_{\nu \beta} * F^{\alpha \beta} .
$$

The point is that any transformation must be represented in terms of the independent field variables, which Eq. (7) cannot as it is most easily seen by observing that $d^{*} F$ (unlike $d F$ ) does not vanish identically. On the other hand, an explicit generator of the rotation (4) or (5) does exist [1]. ${ }^{2}$ In the $(\mathbf{Z}, \mathbf{A})$ language, it takes the particularly elegant "topological," metric-independent form

\footnotetext{
${ }^{2}$ This is not always the case: for the scalar field in two dimensions, for example, the duality is on $\partial_{\mu} \phi \rightarrow \epsilon_{\mu \nu} \partial^{\nu} \phi$, or in canonical language, on rotation of the canonical momentum to $\phi$ and its gradient, but there is no generator of the equivalent of Eq. (4). Similar difficulties arise for $2 k$-form gauge fields in $2+4 k$ dimensions. While the energy density is invariant under duality rotations, the kinetic term is not. Thus, duality rotations are not canonical transformations in those cases.
}

$$
\begin{aligned}
-2 G & =C S(\mathbf{Z})+C S(\mathbf{A}) \\
& =\int d^{3} x \epsilon^{i j k} Z_{i} \partial_{j} Z_{k}+\int d^{3} x \epsilon^{i j k} A_{i} \partial_{j} A_{k} .
\end{aligned}
$$

We now turn to the black hole case and include electric and magnetic sources. To stick to the problem of interest in [3], where only the exterior solution is considered, one can still work with the sourcefree Maxwell equations but one must allow for nonvanishing electric and magnetic fluxes at infinity. This is possible because the spatial sections $\Sigma$ have a hole. There are thus two-dimensional surfaces that are not contractible to a point, namely, the surfaces surrounding the hole (we assume for simplicity a single black hole but the analysis can straightforwardly be extended to the multiblack-hole case).

In the presence of a nonvanishing magnetic flux, the magnetic field is given by the expression

$$
B^{i}=\epsilon^{i j k} \partial_{j} A_{k}+B_{S}^{i}
$$

where $B_{S}^{i}$ is a fixed field that carries the magnetic flux,

$$
\oint_{S_{\infty}^{2}} B_{S}^{i} d S_{i}=4 \pi \mu
$$

and where $B_{T}^{i}=\epsilon^{i j k} \partial_{j} A_{k}$ is the transverse part of $B^{i}$,

$$
\partial_{i} B_{T}^{i}=0, \oint_{S_{\infty}^{2}} B_{T}^{i} d S_{i}=0 .
$$

Following Dirac, we can take $B_{S}^{i}$ to be entirely localized on a string running from the source hole to infinity, say along the positive $z$-axis $\theta=0$. We shall not need the explicit form of $B_{S}^{i}$ in the sequel, but only to remember that for a given magnetic charge $\mu, B_{S}^{i}$ is completely fixed and hence is not a field to be varied in the action. The only dynamical components of the magnetic field $B^{i}$ are still the transverse ones, i.e., $A_{i}$.

One can also decompose the electric field as

$$
E^{i}=E_{T}^{i}+E_{L}^{i}
$$

where the longitudinal part carries all the electric flux

$$
\oint_{S_{\infty}^{2}} E_{L}^{i} d S_{i}=4 \pi e,
$$

and the transverse field obeys

$$
\partial_{i} E_{T}^{i}=0, \oint_{S_{\infty}^{2}} E_{T}^{i} d S_{i}=0
$$

and can thus again be written as $E_{T}^{i}=\epsilon^{i j k} \partial_{j} Z_{k}$ for some $Z_{k}$. Given the electric charge $e$, the longitudinal electric field is completely determined if we impose in addition, say, that it be spherically symmetric. As we have done above, we shall work with a variational principle in which we have solved Gauss's law and in which the competing histories

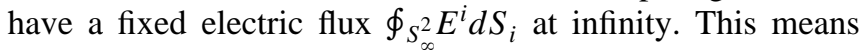
that the longitudinal electric field is completely frozen and that only the tranverse components $E_{T}^{i}$ or $Z^{i}$ are dynamical, as for the magnetic field. 
In order to discuss duality, it is convenient to treat the nondynamical components of $E^{i}$ and $B^{i}$ symmetrically. To that end, one may either redefine $B_{S}^{i}$ by adding to it an appropriate transverse part so that it shares the spherical symmetry of $E_{L}^{i}$, or one may redefine $E_{L}^{i}$ by adding to it an appropriate transverse part so that it is entirely localized on the string. Both choices (or, actually, any other intermediate choice) are acceptable here. For concreteness we may take the first choice; the fields then have no string singularity.

The Maxwell action for the problem at hand takes exactly the same form as Eq. (1), but with $E^{i}$ and $B^{i}$ now the total electric and magnetic fields. Since $E_{L}^{i}$ may be taken to be time independent (the electric charge is constant), one may replace $E^{i}$ by $E_{T}^{i}$ in the kinetic term of Eq. (1), yielding as alternative action

$$
\begin{aligned}
I_{M}^{e, \mu}\left[\mathbf{E}_{T}, \mathbf{A}\right]= & -\int d^{4} x\left[E_{T}^{i} \dot{A}_{i}+\frac{1}{2} N g^{-1 / 2} g_{i j}\left(E^{i} E^{j}+B^{i} B^{j}\right)\right. \\
& \left.-\epsilon_{i j k} N^{i} E^{j} B^{k}\right] .
\end{aligned}
$$

This amounts to dropping a total time derivative-equal to zero for periodic boundary conditions-and shows explicitly that the kinetic term is purely transverse. The action equation (15) involves the parameters $e$ and $\mu$; that is, one has a distinct variational principle for each choice of $e$ and $\mu$, as we have indicated.

To discuss the surface terms that arise in the variation of the action, one must supplement the asymptotic behavior of the fields at infinity by conditions at the horizon. These are especially obvious in the Euclidean continuation, where time becomes an angular variable with the horizon sitting at the origin of the corresponding polar coordinate system. Regularity then requires that $V \equiv A_{0}$ and the time derivatives $\dot{A}_{i}, \dot{E}^{i}$ all vanish at the horizon. We assume these conditions to be fulfilled throughout.

Consider now a duality rotation (4) or (5) acting on the transverse, dynamical variables $A_{i}$ (or $B_{T}^{i}$ ) and $E_{T}^{i}$. Just as in the sourceless case, the kinetic term of Eq. (15) is invariant under this transformation: it is the same kinetic term and the transformation law is the same; the surface term at the horizon in the variation vanishes because $\dot{A}_{i}=0$ and $\dot{Z}_{i}=0$ there. Thus, if we also rotate the (nondynamical) components of the electric and magnetic fields in the same way, that is, if we relabel the external parameters $e, \mu$ by the same transformation as in Eq. (4),

$$
\left(\begin{array}{l}
e^{\prime} \\
\mu^{\prime}
\end{array}\right)=R\left(\begin{array}{l}
e \\
\mu
\end{array}\right)
$$

the actions $I_{M}^{e, \mu}$ and $I_{M}^{e^{\prime}, \mu^{\prime}}$ are equal since $\mathbf{E}$ and $\mathbf{B}$ enter totally symmetrically in the energy and momentum densities. More explicitly, if we write the longitudinal fields as $B_{L}^{i}=\mu V^{i}, E_{L}^{i}=e V^{i}$, then the relevant terms in Eq. (15) are just

$$
\begin{array}{r}
-\int d^{4} x\left\{N g^{-1 / 2} g_{i j}\left[\left(e E_{T}^{i}+\mu B_{T}^{i}\right) V^{j}+\frac{1}{2}\left(e^{2}+\mu^{2}\right) V^{i} V^{j}\right]\right. \\
\left.-\epsilon_{i j k} N^{i} V^{j}\left(e B_{T}^{k}-\mu E_{T}^{k}\right)\right\} .
\end{array}
$$

For the mixed terms, it is clear that the field transformation (4) is just compensated by the parameter rotation (16) (the relevant terms are just the "dot" and "cross" product of the corresponding "two-vectors"), while the $V V$ term is invariant under it. To put it more formally, the extended duality invariance we have spelled out is

$$
I_{M}^{e, \mu}\left[\mathbf{E}_{T}, \mathbf{A}_{T}\right]=I_{M}^{e^{\prime}, \mu^{\prime}}\left[\mathbf{E}_{T}^{\prime}, \mathbf{A}_{T}^{\prime}\right]
$$

where the primes denote the rotated values.

Equation (18) links two actions parametrized by different values of $(e, \mu)$. In particular, for the black holes without Maxwell excitations, we find

$$
I_{M}^{e, 0}[\mathbf{0 , 0}]=I_{M}^{0, e}[\mathbf{0 , 0}],
$$

as in Eq. (3). This equality is thus not a special artifact, but reflects a general invariance property of the action appropriate to the variational principle considered here, in which the electric and magnetic fluxes are kept fixed. The invariance of the action can be verified along the same lines if one also includes the dilaton field.

S.D. thanks the Erwin Schrödinger Institute and the Institute for Theoretical Physics of Vienna University, and M.H. is grateful to LPTHE (Paris VI and Paris VII) for kind hospitality while this work was completed. The work of S.D. was supported by the National Science Foundation Grant No. PHY-9315811, that of M.H. was partly supported by a research grant from FNRS (Belgium), and that of C.T. by Grant No. 1940203 of FONDECYT (Chile) and by institutional support to the Centro de Estudios Científicos de Santiago provided by SAREC (Sweden) and a group of Chilean private companies (EMPRESAS CMPC, CGE, COPEC, MINERA LA ESCONDIDA, NOVAGAS Transportandores de Chile, ENERSIS, BUSINESS DESIGN ASS., XEROX Chile).
[1] S. Deser and C. Teitelboim, Phys. Rev. D 13, 1592 (1976); S. Deser, J. Phys. A 15, 1053 (1982).

[2] D. I. Olive, "Exact Electromagnetic Duality," Report No. hep-th/9508089 (unpublished).
[3] S. W. Hawking and S. F. Ross, Phys. Rev. D 52, 5865 (1995).

[4] R. Arnowitt, S. Deser, and C. W. Misner, in Gravitation, An Introduction to Current Research, edited by L. Witten (Wiley, New York, 1962). 